VOL. 50 (1994) [81-89]

\title{
TOWARDS A CALCULUS OF ALGORITHMS
}

\author{
M. Bulmer, D. Fearnley-Sander and T. Stokes
}

\begin{abstract}
We develop a generalised polynomial formalism which captures the concept of an algebra of piece-wise defined polynomials. The formalism is based on the Boolean power construction of universal algebra. A generalisation of the theory of substitution homomorphisms is developed. The abstract operation of composition of generalised polynomials in one variable is defined and shown to correspond to function composition.
\end{abstract}

On one level, a polynomial may be viewed as an algorithm, giving a sequence of operations to be performed on a finite set of elements of an algebra. We consider an extension of this notion which also captures the important algorithmic notion of branching. The emphasis is entirely algebraic, and the role of substitution is as significant as for standard polynomials. We refer the reader to Lausch and Nobauer [1] for the background theory of generalised polynomials for single sorted universal algebras. We build on the many sorted case, although the basic concepts are essentially the same. The notion of a many-sorted algebra is a natural generalisation of the notion of a universal algebra, in which more than one carrier set is permitted.

A signature $\Sigma$ is a pair consisting of a set $S$ whose elements are called sorts, and a family of sets $\Sigma_{w, \sigma}$ indexed by $w \in S^{*}$ (the set of strings over $S$ ) and $\sigma \in S$. We usually write $f: w \rightarrow b$ instead of $f \in \Sigma_{w, \sigma}$.

A many sorted algebra $A$ of signature $\Sigma$ is a map that associates with each $\sigma \in S$ a set $A_{\sigma}$, and with each $f: \sigma_{1} \sigma_{2} \cdots \sigma_{n} \rightarrow \sigma \in \Sigma_{w, \sigma}$ a function $f^{A}: A_{\sigma_{1}} \times A_{\sigma_{2}} \times \cdots \times$ $A_{\sigma_{n}} \rightarrow A_{\sigma}$. The image $\varepsilon^{A}$ of the empty string is required to be a 1 -element set. We say that the $A_{\sigma}$ are the carriers of $A$ and the $f^{A}$ are the operations on $A$.

A variety of many-sorted algebras of signature $\Sigma$ is a class of algebras closed under the formation of homomorphic images, subalgebras and products, where each of these notions is the natural analog of the corresponding notion from universal algebra. Alternatively, a variety consists of all the algebras of signature $\Sigma$ satisfying a collection of identities.

Received 30th September, 1993

Copyright Clearance Centre, Inc. Serial-fee code: 0004-9729/94 \$A2.00+0.00. 


\section{BoOlean AFFIne COMBINations}

Let $R$ be a Boolean ring, that is, a ring with additive identity $\emptyset$ and multiplicative identity 1 in which every $x \in R$ satisfies $x^{2}=x$. Let addition be denoted by + and multiplication by juxtaposition. The finite subset $\left\{\alpha_{1}, \alpha_{2}, \ldots, \alpha_{k}\right\}$ of $R$ is a partition of unity if

$$
\begin{array}{ll}
\text { 1. } & \sum_{i=1}^{k} \alpha_{i}=1 \text {; and } \\
\text { 2. } & \alpha_{s} \alpha_{t}=\emptyset \text {, for all } s \neq t .
\end{array}
$$

By the Stone Representation Theorem, every Boolean ring $R$ may be interpreted as a ring of subsets of some set $S$, whence a partition of unity may be viewed as a collection of mutually disjoint subsets of $S$ whose union is all of $S$.

Let $T$ be a set and $R$ a Boolean ring. Let $\langle M(T, R), \oplus, \cdot\rangle$ be the free $R$-module with basis $T$, elements expressed in the form $\sum_{t \in T} \alpha_{t} t$ with all but finitely many $\alpha_{t}$ zero. Define $T^{R}$, the set of Boolean affine combinations over $R$ of elements of $T$, to be the set of elements $\sum_{t \in T} \alpha_{t} t$ of $M(T, R)$ such that $\left\{\alpha_{t}: t \in T\right\}$ is a partition of unity. The set $T$ may be viewed as being embedded in $T^{R}$ by identifying each $t \in T$ with the element $1 \cdot t \in T^{R}$. Interpreting $R$ as a ring of subsets of the set $S$ leads readily to the interpretation of $T^{R}$ as the collection of functions $f: S \rightarrow T$ with finite image such that the inverse image of each $t \in \operatorname{Im}(f)$ is in $R$.

PROPOSITION 1.1. Let $p=\sum_{i=1}^{m} \alpha_{i} t_{i} \in M(T, R)$, with $t_{1}, t_{2}, \ldots, t_{m}$ not necessarily distinct elements of $T$. If $\sum_{i=1}^{m} \alpha_{i}=1$ and $\alpha_{i} \alpha_{j}=\emptyset$ for all $i \neq j$, then $p \in T^{R}$.

PRoOF: Collecting like $t_{i}$ terms does not affect the sum of the coefficients and pairwise products will still be zero because of distributivity.

There is an important combinatorial rule for making new Boolean affine combinations from old ones.

Proposition 1.2. Let $p_{1}, p_{2}, \ldots, p_{k} \in M(T, R)$. If $p_{1}, p_{2}, \ldots, p_{k} \in T^{R}$, and $\left\{\alpha_{1}, \alpha_{2}, \ldots, \alpha_{k}\right\} \subseteq R$ is a partition of unity, then $\sum_{i} \alpha_{i} p_{i} \in T^{R}$.

Proof: Let $p_{i}=\sum_{j} \beta_{i j} t_{i j}$ for each i. Then

$$
\sum_{i} \alpha_{i} p_{i}=\sum_{i} \alpha_{i} \sum_{j} \beta_{i j} t_{i j}=\sum_{i, j} \alpha_{i} \beta_{i j} t_{i j}
$$

with

$$
\sum_{i, j} \alpha_{i} \beta_{i j}=\sum_{i} \alpha_{i} \sum_{j} \beta_{i j}=\sum_{i} \alpha_{i} \cdot 1=1 \text {, }
$$

and

$$
\alpha_{i_{1}} \beta_{i_{1} j_{1}} \alpha_{i_{2}} \beta_{i_{2} j_{2}}=\left(\alpha_{i_{1}} \alpha_{i_{2}}\right)\left(\beta_{i_{1} j_{1}} \beta_{i_{2} j_{2}}\right)=\emptyset
$$


providing either $i_{1} \neq i_{2}$ or $j_{1} \neq j_{2}$. Hence by Proposition 1.1, $\sum_{i} \alpha_{i} p_{i} \in T^{R}$.

We call this process taking the affine combination of the $p_{i}$ with respect to the $\alpha_{i}$. With the above interpretation of $T^{R}$ as functions $S \rightarrow T$, the affine combination of the $p_{i}$ with respect to the $\alpha_{i}$ is the function $S \rightarrow T$ obtained by defining a new function piecewise in terms of the $p_{i}-$ on the domain subset $\alpha_{i}$ of $S$, the function agrees with $p_{i}$. The above proposition shows that if each of the component functions $p_{i}$ is in $T^{R}$, then so is their affine combination with respect to the partition of unity given by the $\alpha_{i}$.

\section{BOOLEAN POWERS}

Let $A$ be a many sorted algebra of signature $\Sigma$ with sorts $S$, lying in the variety $V$. Let $A^{R}$ denote the collection of sets of Boolean affine combinations $\left\{A_{\sigma}^{R}: \sigma \in S\right\}$. For $n>0$, each operation $r: A_{\sigma_{1}} \times A_{\sigma_{2}} \times \cdots \times A_{\sigma_{n}} \rightarrow A_{\sigma}$ on $A$ can be extended to an operation $A_{\sigma_{1}}^{R} \times A_{\sigma_{2}}^{R} \times \cdots \times A_{\sigma_{n}}^{R} \rightarrow A_{\sigma_{0}}^{R}$ on $A^{R}$ by setting, for each $a_{j}=\sum_{i} \alpha_{i j} a_{i j} \in A_{\sigma_{j}}^{R}$, where $j=1,2, \ldots, n$ (without loss of generality),

$$
\begin{aligned}
r\left(\sum_{i_{1}} \alpha_{i_{1} 1} a_{i_{1}}, \sum_{i_{2}} \alpha_{i_{2} 2} a_{i_{2} 2}, \ldots,\right. & \left.\sum_{i_{n}} \alpha_{i_{n} n} a_{i_{n} n}\right) \\
& =\sum_{i_{1}, i_{2}, \ldots, i_{n}} \alpha_{i_{1} 1} \alpha_{i_{2} 2} \cdots \alpha_{i_{n} n} r\left(a_{i_{1} 1}, a_{i_{2}}, \ldots, a_{i_{n} n}\right) .
\end{aligned}
$$

If $n=0$, so that $r$ is a nullary operation on $A$ which chooses a distinguished $a_{0} \in A_{\sigma_{0}}$, let $r$ induce the nullary operation on $A^{R}$ determined by the distinguished element $a_{0} \in A_{\sigma_{0}}^{R}$.

If $R$ is interpreted as a ring of sets on $S$, then $A^{R}$ may be viewed as being a subalgebra of the many sorted algebra of all many sorted functions $f=\left(f_{\sigma}\right)_{\sigma \in S}$ with $f_{\sigma}: S \rightarrow A_{\sigma}$ for each carrier $A_{\sigma}$ of $A$ under the pointwise operations. Hence $A^{R}$ is a many sorted algebra of signature $\Sigma$ in the variety $V$ having carriers the $A_{\sigma}^{R}$ and operations as described above.

We note that if $A$ is simply a semigroup, then $A^{R}$ is a particular subsemigroup of the multiplicative semigroup of the semigroup ring $R[A]$, so the construction of $A^{R}$ for general $A$ may be viewed as a generalisation of a construction based on semigroup rings over Boolean rings.

The extension of $A$ to $A^{R}$ thus defined is nothing but the Boolean Power of $A$ by $R$. Boolean powers are an important tool in universal algebra. See for example Pinus [2], where the Boolean power $A^{R}$ is defined to be the algebra of continuous functions with pointwise operations from the Stone space of the Boolean ring (strictly, Boolean algebra) $R$, and taking values in the algebra $A$ endowed with the discrete topology, in 
the one-sorted case a definition abstractly equivalent to the purely algebraic one given here, as is in essence pointed out in Pinus [2]. The extension to the many sorted case is a straightforward process.

\section{VARIABLES}

$A^{R}$ may be viewed as an abstract algebra of "step functions", or piecewise defined constant functions. However, the more general notion of a piecewise defined algebraic function may be captured by linking the structures of $A$ and $R$. We do this by letting $A$ be an algebra of polynomials in a set of variables $X$ and letting $R$ be a Boolean ring consisting of predicates in the variables $X$.

Let $A$ be an algebra of signature $\Sigma$ in a variety $V$. Let $A[X]$ be the algebra in $V$ freely generated by $A$ and the generators $X_{\sigma}=\left\{x_{1}^{\sigma}, x_{2}^{\sigma}, \ldots, x_{m_{\sigma}}^{\sigma}\right\}$ associated with each carrier $A_{\sigma}$ of $A$, and let $X=\left\{X_{\sigma}: \sigma \in S\right\}$. The carrier $A_{\sigma}[X]$ in $A[X]$ corresponds to $A_{\sigma}$ in $A$ for each $\sigma \in S$. Let $N=\left\{m_{\sigma}: \sigma \in S\right\}$ and denote $\Pi_{\sigma \in S} A_{\sigma}[X]^{m_{\sigma}}$ by $A[X]^{N}$; similarly denote $\Pi_{\sigma \in S} A_{\sigma}^{m_{\sigma}}$ by $A^{N}$.

Let $f(x)=f\left(x_{1}^{\sigma}, x_{2}^{\sigma}, \ldots, x_{m_{\sigma}}^{\sigma}: \sigma \in S\right)$ denote a typical element of $A_{\sigma}[X]$. Each such $f(x)$ induces a function $A[X]^{N} \rightarrow A_{\sigma_{0}}[X]$ defined by substitution, namely, for $q=\left(q_{1}^{\sigma}, q_{2}^{\sigma}, \ldots, q_{m_{\sigma}}^{\sigma}: \sigma \in S\right) \in A[X]^{N}$ define $f(q)=f\left(q_{1}^{\sigma}, q_{2}^{\sigma}, \ldots, q_{m_{\sigma}}^{\sigma}: \sigma \in S\right) \in$ $A_{\sigma_{0}}[X]$. There is an obvious restriction of $f: A^{N} \rightarrow A_{\sigma}$.

We note that essentially all of the definitions and constructions in what follows may just as well be made in terms of the important subalgebra of $A[X], F_{V}[X]$, the free algebra in $V$ on the many sorted variable set $X . A[X]$ may be viewed as a generalisation of the ring of polynomials in several variables over a field, in which each monomial may be multiplied by a field element and each polynomial has a constant term: the structure of $A$ is built into $A[X]$, whereas $F_{V}[X]$ simply reflects the nature of the variety $V$. In viewing $f \in A_{\sigma}[X]$ as a function $A^{N} \rightarrow A_{\sigma}$, greater generality is obtained by considering $A[X]$ rather than $F_{V}[X]$, although it is easy to see that the arguments to follow concerning $\mathrm{A}[\mathrm{X}]$ apply to the smaller algebra $F_{V}[X]$ also.

Let $P$ be a collection of function symbols of type $X$, that is, expressions of the form $\rho(x)=\rho\left(x_{1}^{\sigma}, x_{2}^{\sigma}, \ldots, x_{m_{\sigma}}^{\sigma}: \sigma \in S\right)$. Then the set of algebraic predicates, $P r$, consists of all expressions of the form $\psi\left(q_{1}^{\sigma}, q_{2}^{\sigma}, \ldots, q_{m_{\sigma}}^{\sigma}: \sigma \in S\right)$ for some $\psi \in P$ and $q_{j}^{\sigma} \in A_{\sigma}[X]$. Such an algebraic predicate is itself an expression in the $x_{j}^{\sigma}$ and will be denoted by $r(x)$. Denote by $\left.r(x)\right|_{x \mapsto q}$, or $r(q)$ as convenient, the result of the substitution by each $q_{i}^{\sigma}$ in $q=\left(q_{i}^{\sigma}\right) \in A[X]^{N}$ for each $x_{i}^{\sigma}$ occurring in $r(x) \in P r$. Clearly if $r(x) \in P r$, then $r(q) \in P r$ also.

Let $\mathcal{B}(P r)$ denote the free Boolean ring generated by the elements of $P r$. Then $A[X, P]=A[X]^{B(P r)}$ is the algebra of potential piecewise polynomials (the PPP-algebra) of type $X$ associated with $A$ and $P$. 
For $f(x)=\sum \alpha_{i}(x) f_{i}(x) \in A_{\sigma}[X]^{B(P r)}$ and $q \in A[X]^{N}$, define $f(q)=\sum \alpha_{i}(q) f_{i}(q)$. Note that if $a \in A^{N}, f(a)$ will generally not be an element of $A$; for that to be the case we need a functional interpretation of the elements of $P$.

Let $P^{\prime}$ be a collection of functions $A^{N} \rightarrow \mathbf{Z}_{2}$ such that there is a bijection *: $P \rightarrow P^{\prime}$. We call $*$ an instance of $P$. We may extend $*$ to all of $P r$ as follows: for any $\theta(x)=\rho(f) \in \operatorname{Pr}$, where $\rho \in P$ and $f \in A[X]^{N}$, define $\theta^{*}(x)=\psi^{*}(f)$ by setting

$$
\psi^{*}\left(f_{1}^{\sigma}, f_{2}^{\sigma}, \ldots, f_{m_{\sigma}}^{\sigma}: \sigma \in S\right)(a)=\psi^{*}\left(f_{1}^{\sigma}(a), f_{2}^{\sigma}(a), \ldots, f_{m_{\sigma}}^{\sigma}(a): \sigma \in S\right)
$$

for all $q \in A^{N}$. Notation: $\psi^{*}(f)(a)=\psi^{*}(f(a))$. Let $P r^{\prime}$ be the collection of all such $\theta^{*}$.

Let $\mathcal{B}\left(P r^{\prime}\right)$ denote the ring of functions $A^{N} \rightarrow \mathbf{Z}_{2}$ generated by $P r^{\prime}$ under the pointwise operations of addition and multiplication; then $\mathcal{B}\left(P r^{\prime}\right)$ is a Boolean ring. Because $\mathcal{B}(P r)$ is free, there is a unique homomorphism $\Phi: \mathcal{B}(P r) \rightarrow \mathcal{B}\left(P r^{\prime}\right)$ determined by the bijection *:Pr $\rightarrow P r^{\prime}$; let $\alpha^{*}=\Phi(\alpha)$ for all $\alpha \in \mathcal{B}(P r)$.

The functional view may be taken one step further. Given an instance * of $P$ (and hence of $P r$ ), elements of $A[X]^{B(P r)}$ may be associated with functions $A^{N} \rightarrow A_{j}$ for each $j$ as follows. For $\delta(x)=\sum \alpha_{i}(x) p_{i}(x) \in A_{j}[X]^{B(P r)}$, and $a=\left(a_{i}\right) \in A^{N}$, define $\delta^{*}(x)=\sum \alpha_{i}^{*}(x) p_{i}(x) \in A_{j}[X]^{\mathcal{B}\left(P r^{*}\right)}$, a carrier of $A[X]^{B\left(P r^{*}\right)}$. Define $\delta^{*}: A^{N} \rightarrow A_{\sigma}$ by setting $\delta^{*}(a)=\sum \alpha_{i}^{*}(a) p_{i}(a)$, which may be viewed as an element of $A_{j}^{\mathbf{Z}_{2}}$, that is, essentially an element of $A_{j}$ and hence of $A$.

The reason for the name "potential piecewise polynomial" is now clearer: given an instance $*$ of $P$ and a potential polynomial $\delta(x) \in A[X]^{\mathcal{B}(P r)}$, the function $\delta^{*}(x)$ is evaluated at each point by evaluating a multivariate polynomial. The term "potential" signifies the fact that an instance of $\rho$ must be chosen before $\delta(x)$ becomes an abstract piecewise polynomial.

Let $I_{*}=\left\{r(x) \in \mathcal{B}(P r): r^{*}(a)=\emptyset\right.$ for all $\left.a \in A^{N}\right\}$. It is easily seen that $I_{*} \triangleleft \mathcal{B}(P r)$, that is, $I_{*}$ is a ring ideal of $\mathcal{B}(P r)$.

\section{PROPOSITION 3.1. $I_{*}$ is closed under replacements.}

Proof: For any $i \in I_{*}, i^{*}(a)=\emptyset$ for all $a \in A^{N}$; hence if $q \in A[X]^{N}$ then $i(q)^{*}(a)=i^{*}(q(a))=\emptyset$ since $q(a) \in A^{N}$, whence $i(q) \in I_{*}$.

We observe that $I_{*}=\operatorname{Ker}(\Phi)$, so $\mathcal{B}\left(P r^{\prime}\right) \cong \mathcal{B}(P r) / I_{*}$. Let

$$
\gamma: A[X]^{\mathcal{B}\left(P r^{\prime}\right)} \rightarrow A[X]^{B(P r) / I_{*}}
$$

be the induced canonical isomorphism, with inverse $\tau$.

More generally, let $I$ be any ideal of $\mathcal{B}(P r)$ which is closed under replacements. Let $R=\mathcal{B}(P r) / I$. We note that $A[X]^{R}$ is a homomorphic image of $A[X]^{B(P r)}$, via the mapping taking $\Sigma \alpha_{i} a_{i} \in A[X]^{B(P r)}$ to $\Sigma\left(\alpha_{i}+I\right) a_{i} \in A[X]^{R}$; this is a basic property of Boolean powers. (See Pinus [2] for the single sorted case.) 
Theorem 3.2. Let $q \in A[X]$.

Define $g_{q}^{I}: R \rightarrow R$ by setting $g_{q}^{I}(r(x)+I)=r(q)+I$ for all $r(x) \in \mathcal{B}(P r)$. Then $g_{q}^{I}$ is well defined and is an endomorphism.

Define $h_{q}^{I}: A[X]^{R} \rightarrow A[X]^{R}$ by setting $h_{q}^{I}\left(\Sigma\left(r_{i}+I\right)(x) p_{i}(x)\right)=\Sigma\left(r_{i}+I\right)(q) p_{i}(q)$ for all $\sum r_{i}(x) p_{i}(x) \in A[X]^{B(P r)}$. Then $h_{q}^{I}$ is well defined and is an endomorphism.

Proof: We begin by showing $g_{q}^{I}$ is well defined. Let $r(x) \in \mathcal{B}(P r)$. Let $r_{1}(x) \in$ $\mathcal{B}(P r)$ be an element of the coset $r(x)+I$ with $r_{1}(x) \neq r(x)$. We must show that $r_{1}(q)$ is in the coset $r(q)+I$. Suppose $r_{1}(x)=r(x)+s(x)$, for some $s(x) \in I$. Then $r_{1}(q)=$ $r(q)+s(q)$. But by assumption, $s(q) \in I$ and so $r_{1}(q) \in r(q)+I$. Thus $g_{q}^{I}$ is well defined. Now $g_{q}^{I}\left(\left(r_{1}(x)+I\right)+\left(r_{2}(x)+I\right)\right)=g_{q}^{I}\left(\left(r_{1}(x)+r_{2}(x)\right)+I\right)=\left(r_{1}(q(x))+r_{2}(q(x))\right)+$ $I=\left(r_{1}(q(x))+I\right)+\left(r_{2}(q(x))+I\right)=g_{q}^{I}\left(r_{1}(x)+I\right)+g_{q}^{I}\left(r_{2}(x)+I\right)$, and similarly $g_{q}^{I}\left(\left(r_{1}(x)+I\right)\left(r_{2}(x)+I\right)\right)=g_{q}^{I}\left(r_{1}(x)+I\right) g_{q}^{I}\left(r_{2}(x)+I\right)$. Hence $g_{q}^{I}$ is an endomorphism.

Next we show that the mapping $h_{q}^{I}: A[X]^{R} \rightarrow A[X]^{R}$ defined by $h_{q}^{I}\left(\Sigma\left(r_{i}+I\right)(x) p_{i}(x)\right)=\Sigma\left(r_{i}+I\right)(q) p_{i}(q)$ is well defined. Let $\Sigma s_{i}(x) p_{i}(x)$ be an element of the congruence class $\Sigma\left(r_{i}(x)+I\right) p_{i}(x)$. As before, we must show that $\Sigma s_{i}(q) p_{i}(q)$ is in the congruence class $\Sigma\left(r_{i}+I\right)(q) p_{i}(q)$. Suppose $\Sigma s_{i}(x) p_{i}(x)=$ $\Sigma\left(r_{i}(x)+t_{i}(x)\right) p_{i}(x)$, for some $t_{i} \in I$. Then $\Sigma s_{i}(q) p_{i}(q)=\Sigma\left(r_{i}(q)+t_{i}(q)\right) p_{i}(q)$. But by assumption, each $t_{i}(q) \in I$ and so $\Sigma s_{i}(q) p_{i}(q) \in \Sigma\left(r_{i}(q)+I\right) p_{i}(q)$. Thus $h_{q}^{I}$ is well defined.

Finally, we show that $h_{q}^{I}$ is an endomorphism. Let $\rho: A_{\sigma_{1}} \times A_{\sigma_{2}} \times \cdots \times A_{\sigma_{k}} \rightarrow A_{\sigma_{0}}$ be a $k$-ary operation on $A$ and let $\sum_{j} r_{i j}(x) p_{i j}(x) \in A_{\sigma_{i}}[X]$ for each $i$. Then

$$
\begin{aligned}
& \left.\rho\left(\sum_{j}\left(r_{1 j}(x)+I\right) p_{1 j}(x), \ldots, \sum_{j}\left(r_{n j}(x)+I\right) p_{n j}(x)\right)\right|_{x \mapsto q} \\
& \quad=\left.\Sigma_{j_{1}, \ldots, j_{n}}\left(r_{1, j_{1}}(x)+I\right) \cdots\left(r_{n, j_{n}}(x)+I\right) \rho\left(p_{1, j_{1}}(x), \ldots, p_{n, j_{n}}(x)\right)\right|_{x \mapsto q} \\
& \quad=\Sigma_{j_{1}, \ldots, j_{n}}\left(r_{1, j_{1}}(q)+I\right) \cdots\left(r_{n, j_{n}}(q)+I\right) \rho\left(p_{1, j_{1}}(q), \ldots, p_{n, j_{n}}(q)\right), \\
& \quad=\left.\rho\left(\sum_{j}\left(r_{1 j}(q)+I\right) p_{1 j}(q), \ldots, \sum_{j}\left(r_{n j}(q)+I\right) p_{n j}(q)\right)\right|_{x \mapsto q}
\end{aligned}
$$

since substitution is an endomorphism of $R$ as shown above,

Let $I=\{0\}$ in the above theorem; then we may refer to the substitution in any $f(x) \in A[X]^{B\left(P_{r}\right)}$ of a tuple $q$ of elements of $A[X]$, and we denote such a substitution by $f(q)$.

Corollary 3.3. For $q \in A[X]^{N}$, define $\theta_{q}: A[X, P] \rightarrow A[X, P]$ by setting $\theta_{q}(f(x))=f(q)$. Then $\theta_{q}$ is an endomorphism. 
Corollary 3.4. Let $*$ be an instance of $P$. For $f^{*}(x) \in A[X]^{B\left(P r^{\prime}\right)}$ and $q \in A[X]^{N}, s_{q}: A[X]^{B\left(P r^{\prime}\right)} \rightarrow A[X]^{B\left(P r^{\prime}\right)}$ given by $s_{q}\left(f^{*}(x)\right)=f^{*}(q(x))$ is an endomorphism.

Proof: Let $f^{*}(x)=\sum_{i} \alpha_{i}^{*}(x) p_{i}(x)$. Then, with $\gamma$ and $\tau$ defined after the proof of Proposition 3.1 and $\theta_{q}$ as in Corollary 3.3, we have

$$
\begin{aligned}
\left(\tau \circ \theta_{q} \circ \gamma\right)\left(f^{*}(x)\right) & =\tau\left(\theta_{q}\left(\gamma\left(\sum_{i} \alpha_{i}^{*}(x) p_{i}(x)\right)\right)\right) \\
& =\tau\left(\theta_{q}\left(\sum_{i}\left(\alpha_{i}(x)+I_{*}\right) p_{i}(x)\right)\right) \\
& =\tau\left(\sum_{i}\left(\alpha_{i}(q)+I_{*}\right) p_{i}(q)\right) \\
& =\sum_{i} \alpha_{i}^{*} q(x) p_{i}(q(x)) \\
& =f^{*}(q(x)),
\end{aligned}
$$

so $s_{q}=\tau \circ \theta_{q} \circ \gamma$, which is therefore an endomorphism of $A[X]^{\mathcal{B}\left(P r^{\prime}\right)}$.

In particular, if $a \in A^{N}$ and $f(x) \in A_{\sigma_{i}}[X, P]$ then $f^{*}(a) \in A_{\sigma_{i}}$, and the restriction mapping $s_{a}: A[X] \rightarrow A_{\sigma_{i}}$ is a homomorphism, the Evaluation Homomorphism associated with $a$, a generalisation of the corresponding idea from the theory of polynomials over a field.

\section{Composition}

Here we focus on the one variable, single sorted case because the formulation is rather clearer. There is a fairly obvious extension of the ideas discussed here to many variable many sorted situations, although the notion of composition of functions (which we are modelling) is most natural in the single sorted case.

Suppose $A$ is a single sorted (or universal) algebra. Let $X=\{x\}$. We adopt the notation $A[x]$ for $A[X]$. Elements of $A[x]^{B\left(P r^{\prime}\right)}$ may be viewed as operators on $A$.

For $f(x)=\sum_{i} \alpha_{i}(x) p_{i}(x)$ and $g(x)=\sum_{i} \beta_{i}(x) q_{i}(x)$ in $A[x]^{B(P r)}$, define

$$
(g \circ f)(x)=\sum_{i, j} \alpha_{i}(x) \beta_{j}\left(p_{i}(x)\right) q_{j}\left(p_{i}(x)\right)
$$

We call $(g \circ f)(x)$ the composition of $f(x)$ and $g(x)$, for the following reason: 
Proposition 4.1. Let $*$ be an instance of $P$, with $f(x), g(x) \in A[x]^{\mathcal{B ( P r})}$. Then $(g \circ f)^{*}(a)=g^{*}\left(f^{*}(a)\right)$ for all $a \in A$.

Proof: Let $f(x)=\sum_{i} \alpha_{i}(x) p_{i}(x)$ and $g(x)=\sum_{i} \beta_{i}(x) q_{i}(x)$. The $\alpha_{i}(x)$ are a partition of unity, so $\alpha_{i}(x) \alpha_{j}(x)=\emptyset$ and $\sum_{i} \alpha_{i}(x)=1$, so for $a \in A$, there is a unique $j$ such that $\alpha_{j}^{*}(a)=1$, with $\alpha_{k}^{*}(a)=\emptyset$ for all $k \neq i$. Hence $f^{*}(a)=p_{j}(a)$, so $g^{*}\left(f^{*}(a)\right)=g^{*}\left(p_{j}(a)\right)$. Repeating the argument for $f(x)$, there is a unique $k$ such that $\beta_{k}^{*}\left(p_{j}(a)\right)=1$, with $\beta_{i}^{*}\left(p_{j}(a)\right)=\emptyset$ for all $i \neq k$. Hence $g^{*}\left(f^{*}(a)\right)=q_{k}\left(p_{j}(a)\right)$.

On the other hand, $(g \circ f)(x)=\sum_{i, l} \alpha_{i}(x) \beta_{l}\left(p_{i}(x)\right) q_{l}\left(p_{i}(x)\right)$. So

$$
\begin{aligned}
(g \circ f)^{*}(a) & =\sum_{i, l} \alpha_{i}^{*}(a) \beta_{l}^{*}\left(p_{j}(a)\right) q_{l}\left(p_{i}(a)\right) \\
& =\sum_{l} \alpha_{j}^{*}(a) \beta_{l}^{*}\left(p_{j}(a)\right) q_{l}\left(p_{j}(a)\right) \\
& =\alpha_{j}^{*}(a) \beta_{k}^{*}\left(p_{j}(a)\right) q_{k}\left(p_{j}(a)\right) \\
& =1 \cdot 1 \cdot q_{k}\left(p_{j}(a)\right) \\
& =g^{*}\left(f^{*}(a)\right) .
\end{aligned}
$$

The analogous formula for compositions of functions applies to elements of $A[x]^{B\left(P r^{\prime}\right)}$.

Corollary 4.2. Let $f^{*}(x), g^{*}(x) \in A[x]^{B\left(P r^{\prime}\right)}$. Define $g^{*} \circ f^{*}=(g \circ f)^{*}$. Then $\left(g^{*} \circ f^{*}\right)(a)=g^{*}\left(f^{*}(a)\right)$.

PROOF: We show that $g^{*} \circ f^{*}=(g \circ f)^{*}$ is well defined on $A[x]^{B\left(P r^{\prime}\right)}$. Let $f_{1}, g_{1} \in A[x]^{B(P r)}$ be such that $f_{1}^{*}=f^{*}$ and $g_{1}^{*}=g^{*}$. Then, for all $a \in A$,

$$
(g \circ f)^{*}(a)=g^{*}\left(f^{*}(a)\right)=g_{1}^{*}\left(f_{1}^{*}(a)\right)=\left(g_{1} \circ f_{1}\right)^{*}(a)
$$

by Proposition 4.1, so $(g \circ f)^{*}=\left(g_{1} \circ f_{1}\right)^{*}$ and so $g^{*} \circ f^{*}=(g \circ f)^{*}$ is well defined, and

$$
\left(g^{*} \circ f^{*}\right)(a)=(g \circ f)^{*}(a)=g^{*}\left(f^{*}(a)\right)
$$

by Proposition 4.1.

Much of the motivation for the work of this paper lies in the authors' desire to extend the notion of polynomial so that it can better capture the kinds of functions which are in practice computable. The next obvious extension of the polynomial idea would permit the definition of recursively defined functions. 


\section{REFERENCES}

[1] H. Lausch and W. Nobauer, Algebra of polynomials, North-Holland mathematical library 5 (North-Holland, Amsterdam, 1973).

[2] A.G. Pinus, 'Boolean Constructions in Universal Algebra', Russian Math. Surveys 47 (1992), 157-198.

Department of Mathematics

University of Tasmania

Hobart, Tas 7000 Australia 